Wright State University

CORE Scholar

6-1-2012

\title{
Extraordinary Optical Transmission and Extinction in a Terahertz Wire-Grid Polarizer
}

\author{
J. S. Cetnar \\ J. R. Middendorf \\ Elliott R. Brown \\ Wright State University - Main Campus, elliott.brown@wright.edu
}

Follow this and additional works at: https://corescholar.libraries.wright.edu/physics

Part of the Physics Commons

\section{Repository Citation}

Cetnar, J. S., Middendorf, J. R., \& Brown, E. R. (2012). Extraordinary Optical Transmission and Extinction in a Terahertz Wire-Grid Polarizer. Applied Physics Letters, 100 (23), 231912.

https://corescholar.libraries.wright.edu/physics/102

This Article is brought to you for free and open access by the Physics at CORE Scholar. It has been accepted for inclusion in Physics Faculty Publications by an authorized administrator of CORE Scholar. For more information, please contact library-corescholar@wright.edu. 


\title{
Extraordinary optical transmission and extinction in a Terahertz wire-grid polarizer
}

\author{
John S. Cetnar, ${ }^{1}$ John R. Middendorf, ${ }^{1}$ and Elliott R. Brown ${ }^{1,2}$ \\ ${ }_{1}^{1}$ Department of Electrical Engineering, Wright State University, Dayton, Ohio 45435, USA \\ ${ }^{2}$ Department of Physics, Wright State University, Dayton, Ohio 45435, USA
}

(Received 6 April 2012; accepted 16 May 2012; published online 8 June 2012)

\begin{abstract}
A $\mathrm{THz}$ wire grid polarizer is simulated and demonstrated consisting of $40-\mu \mathrm{m}$ periodic aluminum strips mounted on a polycarbonate substrate with a variable metal-to-gap ratio. Full-wave numerical simulations were performed from $100 \mathrm{GHz}$ to $550 \mathrm{GHz}$ predicting that the transmission in perpendicular (parallel) polarization is much higher (lower) than that predicted by geometric optics, leading to a very high extinction ratio of $\sim 60 \mathrm{~dB}$ between 100 and $550 \mathrm{GHz}$ when the gaps become very small $(<5 \mu \mathrm{m})$. This behavior is confirmed qualitatively in experiments between 100 and $530 \mathrm{GHz}$ where extinction ratios exceeding $40 \mathrm{~dB}$ are achieved. These results are explained physically as an electromagnetic concentration effect in the gaps consistent with plasmonic-like behavior. The effect depends critically on gap width and weakly on frequency. (c) 2012 American Institute of Physics. [http://dx.doi.org/10.1063/1.4724315]
\end{abstract}

There have been many recent studies on the phenomena of extraordinary optical transmission (EOT). ${ }^{1-4}$ These studies concern optical transmission through sub-wavelength apertures in thin metal conductive sheets. The apertures are usually circular and are periodically arranged on a geometric grid. Furthermore, in almost all of these studies the radiation is in the optical and/or near-infrared regions. The physical mechanism explaining EOT in these studies is the resonant coupling of electromagnetic radiation in the air with surface plasmon polaritons (SPPs) (i.e., surface plasmons) on the conductor surface. EOT has been seen in the microwave, millimeter wave, and sub-millimeter wave $(\mathrm{THz})$ regions ${ }^{5,6}$ as well. However, in these regions the mechanism for EOT is not SPP coupling, strictly speaking. ${ }^{7}$ In metal conductors, a high concentration of free electrons ensures that well-confined SPPs can exist only for frequencies relatively close to the conductor's plasma frequency $\omega_{\mathrm{p}}$. Thus, SPPs are supported in the visible and near-infrared regions. In order to have SPP propagation on the air-conductor interface, the SPP propagation constant must be larger than the electromagnetic (EM) wavevector, $\beta>\mathrm{k}_{0}$. This condition leads to decaying fields perpendicular to the air-metal interface. The amount of the confinement increases with increasing $\beta$ and decreases significantly for $\omega \ll \omega_{\mathrm{p}}$, where $\beta$ asymptotically approaches $\mathrm{k}_{0}$. In the $\mathrm{THz}$ regions, the SPP propagation constant $\beta$ is approximate to $\mathrm{k}_{0}\left(\beta \approx \mathrm{k}_{0}\right)$. This is because there is a large real part of the complex permittivity $\left(\epsilon \approx 10^{5}\right)$ for metal conductors at these frequencies. This in turn prevents significant field penetration into the conductor and thus the SPPs are highly delocalized. In effect, SPPs at THz frequencies are not confined to the conductor's surface and radiate away.

However, at $\mathrm{THz}$ and lower frequencies SPP-like behavior can be created by an effective-medium phenomenon (i.e., "spoof surface plasmons" ${ }^{\text {) }}$. A grid of periodically spaced apertures in a metal film can support spoof SPPs when $\mathrm{d}<\mathrm{a}$ $\ll \lambda$ ( $\mathrm{d}=$ aperture diameter, $\mathrm{a}=$ grid period, and $\lambda=$ radiation wavelength). This is essentially the same mechanism that produces the effects seen in metamaterials, and has been described in circular-hole arrays. ${ }^{8}$ In this paper, we show that spoof-SPPs can be extended to include long, narrow slots such as those that occur in a wire-grid polarizer consisting of a strip array on an insulating substrate. This is justified physically by surface currents that flow into and around the gaps and support electric and magnetic dipoles. ${ }^{9}$ When the gaps become narrow they concentrate electromagnetic energy incident from one side and re-radiate it to the other, yielding an EOT effect. This is proven by numerical simulation and experiment.

Our polarizer structure consists of an array of aluminum strips having width w and separation (gap) d patterned on an insulating substrate of polycarbonate-a plastic with high RF transparency which can be made flat and smooth enough for micron-scale lithographic fabrication. Our objective was to study this basic design to large values of fill factor, $\mathrm{FF}=\mathrm{w} /(\mathrm{w}+\mathrm{d})>90 \%$, in hopes of utilizing spoof-SPP behavior. The period $(\mathrm{w}+\mathrm{d})$ was fixed at $40 \mu \mathrm{m}$, and the thickness at $0.2 \mu \mathrm{m}$ to match the experimental value determined by evaporation. Millimeter-wave and $\mathrm{THz}$ strip-onsubstrate polarizers have been developed previously ${ }^{10-13}$ but not at the large fill factors ( $\mathrm{FF} \geq 95 \%$ ) investigated here.

The numerical simulations were carried out by a commercial full-wave finite-element tool, high frequency structure simulator (HFSS). The power transmissivity (T) was calculated for incident radiation polarized perpendicular (S) and parallel (P) to the strip axis, and from $100 \mathrm{GHz}$ to $550 \mathrm{GHz}$ in steps of $1 \mathrm{GHz}$ across a range of fill factors. The fill factor was varied by changing the strip width from 0 to $39.9 \mu \mathrm{m}$. Note that the geometries are all sub-wavelength relative to the range of simulated frequencies. The geometry was simulated at the unit cell level, shown in Fig. 1(a). Periodic boundary conditions, known as master/slave boundary conditions in HFSS, were applied. Floquet ports were used to excite the structure with electromagnetic radiation. For all simulations, the incoming radiation was at normal incidence. In addition, the electric and magnetic fields were calculated throughout the simulation volume. This allowed for the viewing and analysis of field strengths and directions at critical locations in the simulation space, particularly in the air 


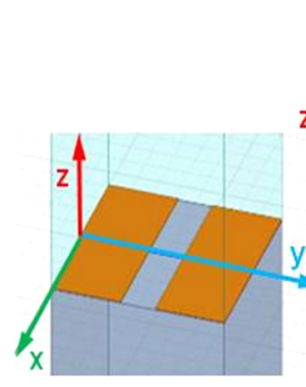

(a)

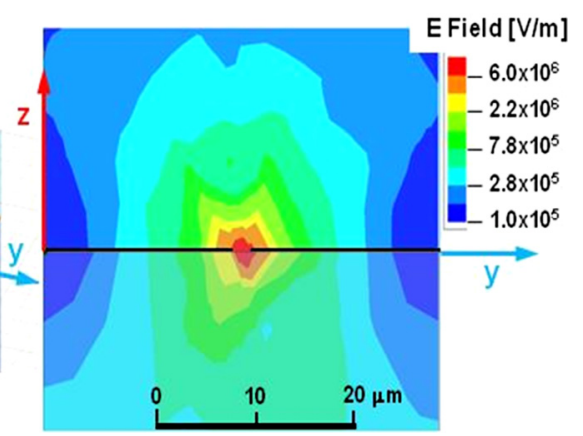

(b)

FIG. 1. (a) $\mathrm{THz}$ wire grid polarizer unit cell geometry for $80 \%$ fill factor. (b) Side view of electric field magnitude (color code) in the gap region for a $95 \%$ fill factor at $530 \mathrm{GHz}$. Note the concentration of the field between the gaps. The slight asymmetry in the electric field strength with respect to the gap bisector is associated with asymmetric meshing in the FEM algorithm used in the calculation.

gaps between the wires. To enhance the accuracy, losses in the aluminum were accounted for using a look-up table for the metal dielectric function based on the Drudian model with momentum relaxation time $\tau=1 \times 10^{-14} \mathrm{~s}$. Absorptive losses in the polycarbonate were also simulated using a separate look-up table containing the frequency-dependent dielectric function derived from broadband transmission data taken with a $\mathrm{THz}$ photomixing spectrometer.

The $\mathrm{T}$ values from $100 \mathrm{GHz}$ to $550 \mathrm{GHz}$ were computed for a wide variety of fill factors ranging from $0 \%$ to $99.75 \%$,

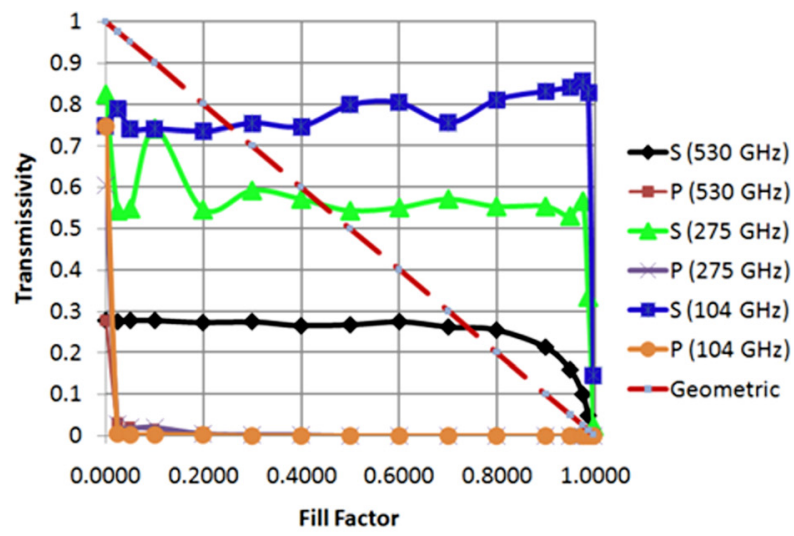

(a)

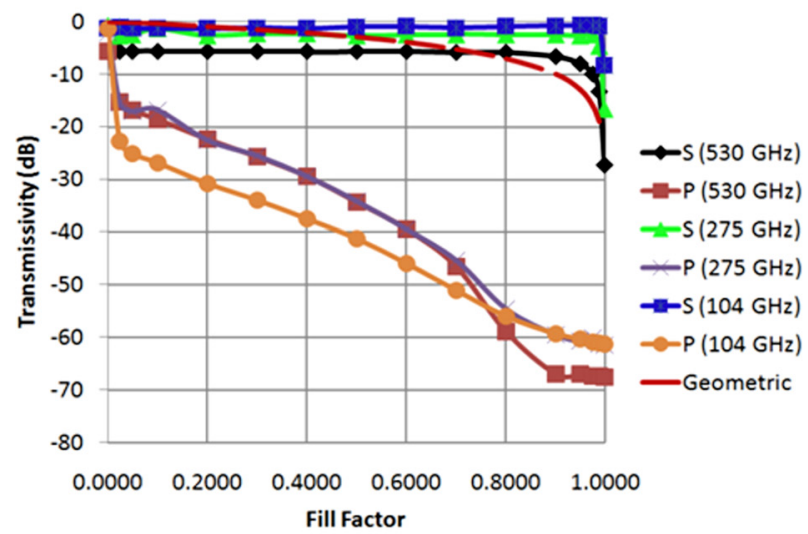

(b)

FIG. 2. (a) Transmissivity versus fill factor for $104 \mathrm{GHz}, 275 \mathrm{GHz}$, and $530 \mathrm{GHz}$. The dashed line is the prediction from geometric optics. (b) Expanded view of (a) plotted in $\mathrm{dB}$ units on vertical axis to see the large difference between $\mathrm{S}$ and $\mathrm{P}$ polarizations. and are plotted on a linear and log scale in Figs. 2(a) and 2(b), respectively. For wire grid polarizers, the insertion loss $\left[\mathrm{IL}=10^{*} \log _{10}(\mathrm{~T})\right]$ and the extinction ratio (ER) are the two most important figures-of-merit. ER is the ratio of the P-polarized $\mathrm{T}$ to the S-polarized T, and is plotted vs. fill factor in Fig. 3(a). As can be seen in Figs. 2(a) and 2(b), the S-polarized transmissivity at each frequency is relatively flat with fill factor up to FF 98\% and then drops precipitously. In contrast, the $\mathrm{P}$ polarized $\mathrm{T}$ drops steadily with FF. Therefore, as shown in Fig. 3(a), the ER increases with increasing fill factor up to a peak of about $60 \mathrm{~dB}$ at an FF between 90 and $100 \%$. The exploded view in Fig. 3(b) shows that the peak fill factor decreases with increasing frequency. Beyond each respective peak both the S-polarized T and the ER drop precipitously, indicative of a critical effect. This makes sense physically because the S-polarized radiation is expected to display the strongest concentration effect by driving surface currents into the gap, not parallel to it. It is also consistent with the great enhancement of the S-polarized transmissivity above the prediction of geometric optics [dashed line in Fig. 2(a)], which justifies the label "EOT." However, note that this optical enhancement only occurs beyond a threshold fill factor that rises with increasing frequency: $\mathrm{FF}=25 \%$ for $104 \mathrm{GHz}, 45 \%$ for $275 \mathrm{GHz}$, and $75 \%$ for $530 \mathrm{GHz}$. This is caused by the continuous drop in $\mathrm{T}$ with frequency displayed in Fig. 2(a), independent of fill factor, and associated with absorptive loss in the polycarbonate substrate.

A microscopic justification for the EOT is provided by the electric-field magnitude computed by the full-wave

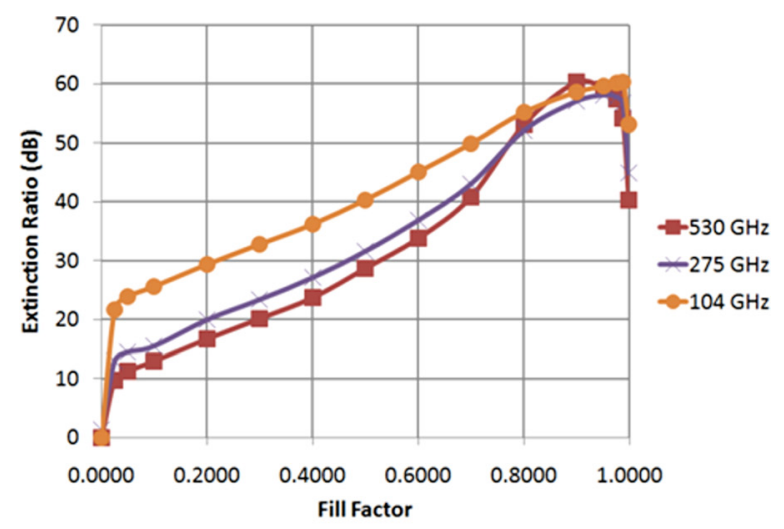

(a)

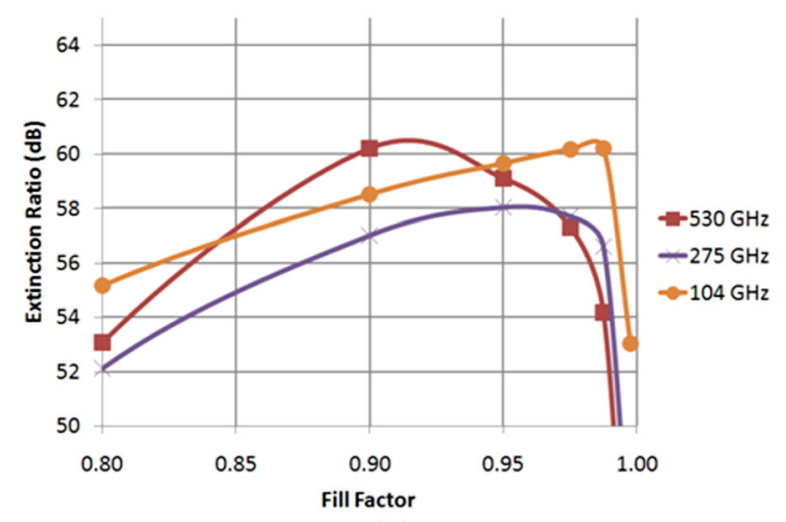

(b)

FIG. 3. (a) Extinction ratio (dB) versus fill factor for $104 \mathrm{GHz}, 275 \mathrm{GHz}$, and $530 \mathrm{GHz}$. (b) Exploded view of (a) in the region of the extinction-ratio peak. 


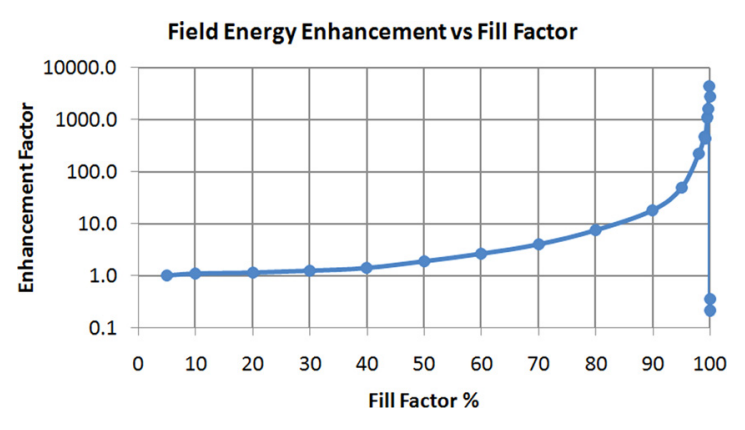

FIG. 4. Enhancement factor versus fill factor at $530 \mathrm{GHz}$.

simulator. An example of the field magnitude for a $95 \%$ fill factor at $530 \mathrm{GHz}$ is displayed in Fig. 1(b). In addition, the enhancement of the field energy at $20 \mathrm{~nm}$ above the gap for $530 \mathrm{GHz}$ was calculated. The FFs ranged from 5\% to $100 \%$. A plot of the enhancement is shown in Fig. 4. Note the very high enhancement at high FFs. This is due to the very high energy densities achieved in the gap at high FFs.

To investigate the predicted behavior experimentally, two wire-grid polarizers were fabricated on polycarbonate substrates with aluminum strips and fill factors of 80 and 95\%. Fig. 5 shows one of the wire grid polarizer samples mounted in an optical rotation stage. Measurements of the IL and ER were carried out at spot frequencies of 104, 275, and $530 \mathrm{GHz}$ using solid-state oscillators coupled to free space with linearly-polarized horn antennas, and waveguidemounted zero-bias Schottky rectifiers also coupled through horn antennas. The experimental results for ER and IL are plotted in Figs. 6(a) and 6(b), respectively. Consistent qualitatively with simulation, the ER in Fig. 6(a) increases with fill factor at each of the three frequencies, and also increases with frequency at a given fill factor. The experimental IL in Fig. 5(b) increases rather fast with frequency at both fill factors consistent with the rapid increase in absorption coefficient in the polycarbonate substrates above $\sim 300 \mathrm{GHz}$. Polycarbonate is clearly not the best choice of polarizer substrate in the $\mathrm{THz}$ region above $\sim 500 \mathrm{GHz}$, but is an excellent choice in the upper mm-wave region below $300 \mathrm{GHz}$ including the increasingly popular W-band $(75-110 \mathrm{GHz})$.

Quantitatively the experimental ER data is lower than the simulated values by roughly $20 \mathrm{~dB}$, depending on fill factor and frequency. This is not yet understood but might be attributed to materials effects such as excess sheet resistance

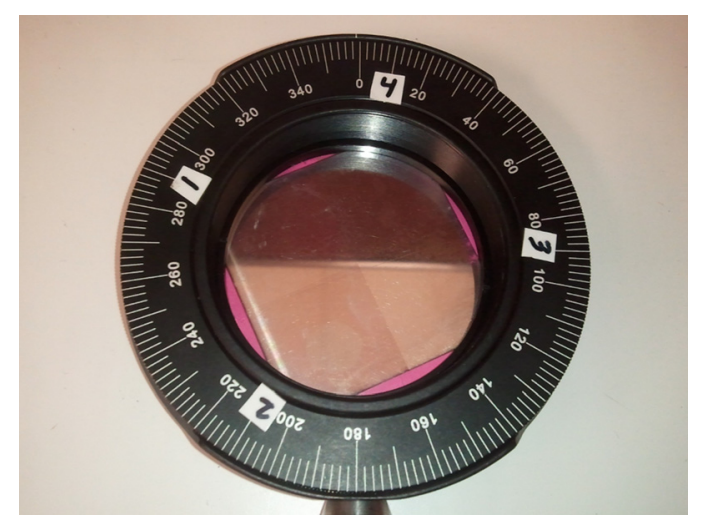

FIG. 5. Fabricated wire grid polarizer mounted in an optical rotation stage.

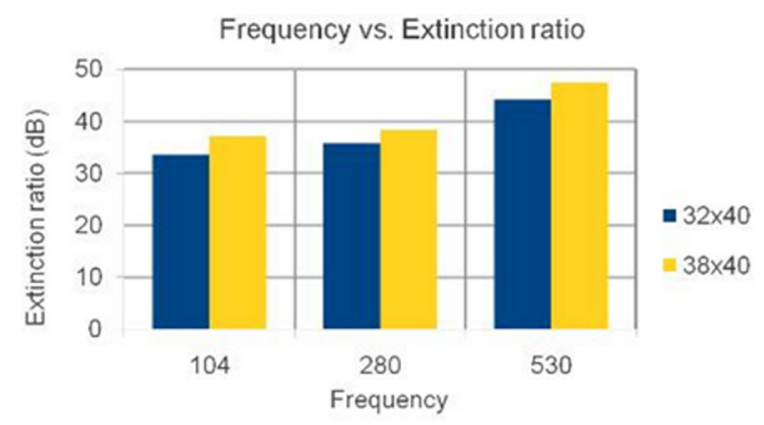

(a)

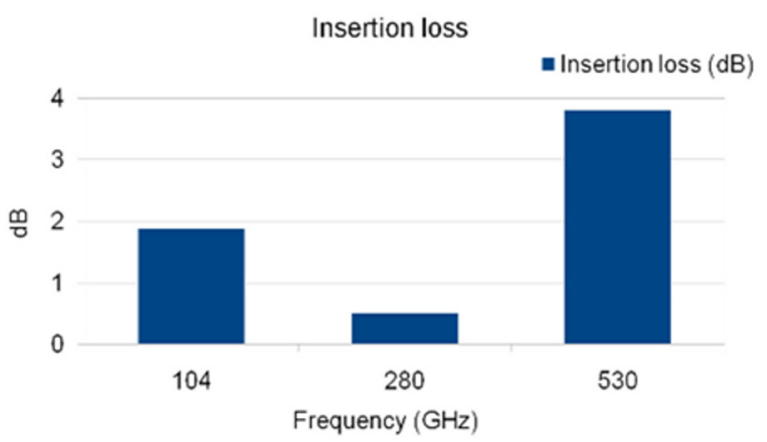

(b)

FIG. 6. (a) The experimental extinction ratio of two different fill factors measured at three spot frequencies. (b) Fill factor vs. average extinction ratio. The trend here matches the simulated results.

in the aluminum strips used for the present structures. Further investigation is necessary.

In conclusion, EOT has been seen in a wire grid polarizer of sub-wavelength periodicity in the $\mathrm{THz}$ region using full-wave numerical simulations. The simulations predicted that the extinction ratio of the polarizer would increase with FF reaching a peak between 90 and $98 \%$ and then decrease. The increase is due to the very high concentration of electromagnetic energy achieved in the gap between the polarizer strips at high FFs. The prediction of the numerical simulations was confirmed qualitatively through experiment.

\footnotetext{
${ }^{1}$ W. L. Barnes, A. Dereux, and T. W. Ebbsen, Nature (London), 424, 824 (2003).

${ }^{2}$ T. W. Ebbesen, H. J. Lezec, H. f. Ghaemi, T. Thio, and P. A. Wolff, Nature (London), 391, 667 (1998).

${ }^{3}$ L. Martín-Moreno, F. J. Garcia-Vidal, H. J. Lezec, K. M. Pellerin, T. Thio, J. B. Pendry, and T. W. Ebbesen, Phys. Rev. Lett. 86, 1114 (2001).

${ }^{4}$ M. Palamaru and S. Astilean, J. Optoelectron. Adv. Mater. 1, 35 (1999).

${ }^{5}$ H. Cao and A. Nahata, Opt. Exp. 12, 1004 (2004).

${ }^{6}$ M. Beruete, M. Sorolla, I. Campillo, J. S. Dolado, L. Martin-Marerno, J. Bravo-Abad, and F. J. Garcia-Vidal, IEEE Trans. Antennas Propag. 53, 1897 (2005).

${ }^{7}$ S. Maier, Plasmonics: Fundamentals and Applications (Springer Science+Business Media, New York, 2007).

${ }^{8}$ J. B. Pendry, L. Martín-Moreno, and F.J. Garcia-Vidal, Science 305, 847 (2004).

${ }^{9}$ J. D. Jackson, Classical Elecrodynamics, 3rd ed. (Wiley, 1999).

${ }^{10} \mathrm{P}$. Piksa and S. Zvanovec, in 2010 Proceedings of the Fourth European Conference on Antennas and Propagation (EuCAP), Barcelona, Spain, 2010, p. 1.

${ }^{11}$ I. Yamada, K. Takano, M. Hangyo, M. Saito, and W. Watanabe, Opt. Lett. 34, 274 (2009).

${ }^{12} \mathrm{~K}$. Takano, I. Morimoto, H. Yokoyama, M. Hangyo, in 35th International Conference on Infrared, Millimeter, and Terahertz Waves (IRMMWTHz), Rome, Italy, 2010, pp. 1-2.

${ }^{13}$ L. Zhang, J. H. Teng, H. Tanoto, S. Y. Yew, L. Y. Deng, S. J. Chua, in 35th International Conference on Infrared, Millimeter, and Terahertz Waves (IRMMW-THz), Rome, Italy, 2010, pp. 1-2.
} 